\title{
SUBSISTENCIA EN ISLA NAVARINO. UN ESTUDIO EXPLORATORIO SOBRE LA VARIABILIDAD ESPACIAL EN LA EXPLOTACIÓN DE RECURSOS AL SUR DEL CANAL BEAGLE, CHILE
}

JAVIERA MARDONES B.

\section{RESUMEN}

Se presentan los resultados obtenidos a partir del análisis zooarqueológico de 17 pozos de sondeo ubicados a lo largo de la costa norte de isla Navarino, localidad que no presenta aún estudios relativos a la subsistencia. De manera preliminar se busca entender cómo se relacionan las variables microambientales con el registro zooarqueológico, para así evaluar la subsistencia desde el eje de variación espacial. Se comparan características ambientales asociadas a los sitios con la estructura y composición de los conjuntos zooarqueológicos. Para ello se utilizan medidas de diversidad taxonómica, principalmente la equiparidad y heterogeneidad. Los resultados muestran que algunas variables resultaron de utilidad mientras que otras requieren de mayor información, por lo que las tendencias observadas deberán ser estudiadas a mayor profundidad en el futuro cercano.

PALABRAS CLAVE: microambientes, zooarqueología, equiparidad, adaptación litoral, canal Beagle.

\section{SUBSISTENCE IN NAVARINO ISLAND. AN EXPLORATORY STUDY ON SPATIAL VARIABILITY IN THE EXPLOITATION OF RESOURCES SOUTH OF THE BEAGLE CHANNEL, CHILE}

\begin{abstract}
We present the results obtained from the zooarchaeological analysis of 17 test pits located along the northern coast of Navarino Island, a location that does not present studies related to subsistence. In a preliminary way we seek to understand how to relate the micro-environmental variables with the zooarchaeological record, in order to evaluate subsistence from the axis of spatial variation. Environmental characteristics associated with the sites are compared with the structure and composition of the zooarchaeological assemblages. In order to do this, measures of taxonomic diversity are used, mainly the evenness and heterogeneity. The results show that some variables were useful while others require more information, so the observed trends should be studied in greater depth in the near future.
\end{abstract}

KEY WORDS: microenvironments, zooarchaeology, evenness, littoral adaptation, Beagle channel.

a $\quad>$ javiera.mardonesb@gmail.com 


\section{INTRODUCCIÓN}

La costa sur de Patagonia fue poblada por grupos canoeros como parte de una adaptación litoral que inicia hacia el Holoceno medio, aproximadamente 6.000 años AP (Legoupil \& Fontugne, 1997; Orquera \& Piana, 2009; Orquera et al. 2011; Legoupil et al. 2011; Morello et al. 2012). Esta adaptación litoral consiste en la predominancia de recursos costeros en la dieta y en la presencia de tecnología especializada para generar un aprovechamiento más eficiente de los recursos (Orquera \& Piana, 1999, 2009).

Los estudios en la costa norte del canal Beagle han sido particularmente relevantes para entender este fenómeno (Orquera \& Piana, 1999), y han planteado un modelo de subsistencia a nivel general donde hay una predominancia de pinnípedos como principal presa, complementada con fauna menor y/o de bajo rendimiento: mayoritariamente aves, peces y moluscos (Piana Orquera et al. 2007). En términos temporales se han detectado variaciones en cuanto a la selección de recursos sobre la base de un aumento en la equiparidad (evenness) entre la representación de los diferentes taxones en distintos sitios de la costa norte del canal Beagle (Tivoli \& Zangrando, 2011). Durante el Holoceno medio habría una predominancia de pinnípedos en los conjuntos, mientras que en el Holoceno tardío aumentarían las frecuencias relativas de aves y peces.

El área de estudio corresponde a la costa noroeste de isla Navarino, entre Puerto Navarino y Puerto Williams (Fig. 1). Abarca una franja de 53 km de largo y considera una franja de 60 metros a cada lado (costa-interior) del camino costero (Ruta Y-905), que corre paralelo a las costas del canal Beagle.

En este informe buscamos evaluar la subsistencia desde el eje de variación espacial, considerando que la disponibilidad de fauna varía de acuerdo a los microambientes (Orquera et al. 2011; San Román, 2011; Tivoli, 2010), por lo que la equiparidad de los conjuntos puede asociarse a esta variable. Si bien a lo largo del canal Beagle los mismos recursos son igualmente asequibles, "hay situaciones topográficas que debieron influir sobre la relación de costos y beneficios relacionados con el aprovechamiento alimenticio o tecnológico de diferentes especies" (Orquera \& Piana, 1999, p. 27). Buscamos evaluar la variabilidad espacial en el registro arqueofaunístico de la costa de isla Navarino para complementar las investigaciones más sistemáticas y de larga duración llevadas a cabo en la costa norte del canal Beagle.

Es importante mencionar que no todas las variables identificadas en este trabajo resultaron de utilidad para comprender la relación entre el registro zooarqueológico y los microambientes. Cabe destacar que estos sitios no cuentan con fechados radiocarbónicos hasta el momento, además, el tamaño de la muestra utilizado para este estudio es aún pequeño para saber si las tendencias que se proponen son representativas. Sin embargo, consideramos que el trabajo realizado consiste en una exploración inicial y permite plantear sugerencias que deberán ser estudiadas con mayor profundidad en el futuro.

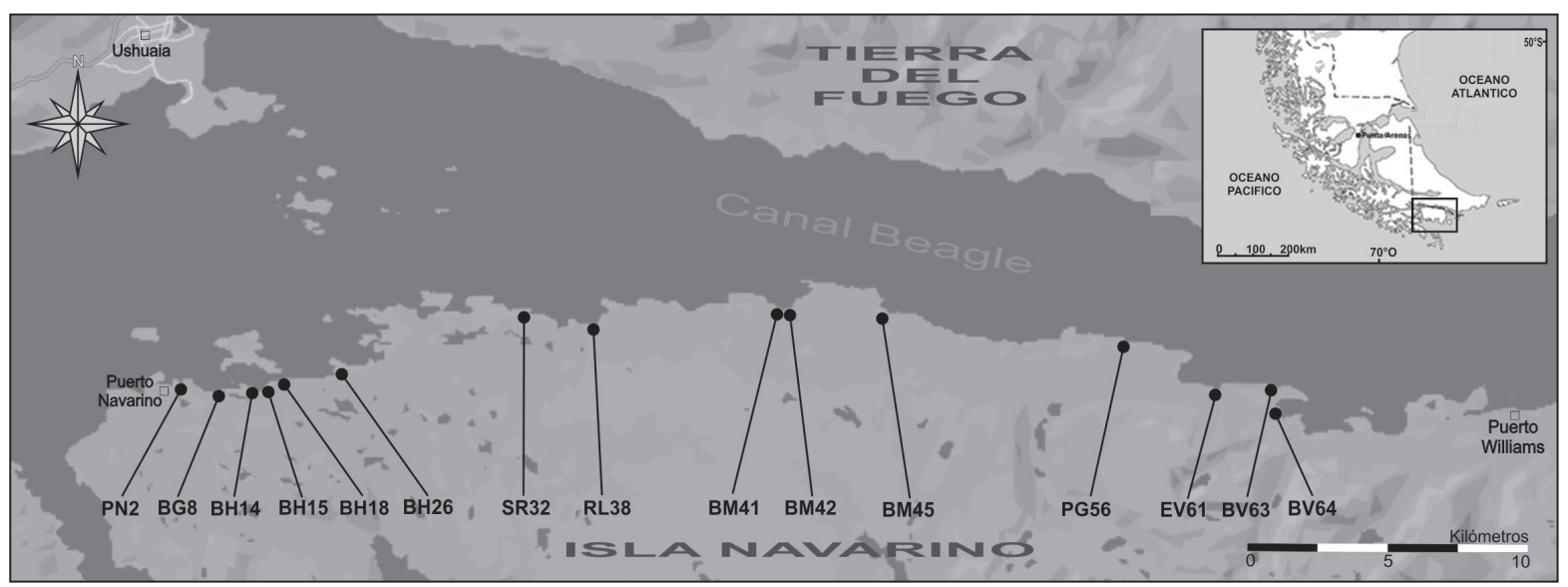

Fig. 1. Mapa del área de estudio y de los sitios trabajados. 


\section{MATERIAL Y MÉTODO}

La muestra consta de un total de 2291 restos faunísticos e incluye mamíferos, aves y peces en buen estado de conservación y completitud. Estos fueron obtenidos desde 17 pozos de sondeo realizados en 15 sitios arqueológicos que presentan diferencias en cuanto a superficie y contexto, representando la variabilidad presente en la zona. Todos los sondeos se presentan como unidades monocomponentes.

Para evaluar la subsistencia desde el eje de variación espacial se realizó en primera instancia una caracterización medioambiental que consistió en la determinación de variables asociadas a los sitios que pudiesen influir en la disponibilidad de los recursos faunísticos: tipo de bahía, protección de la bahía respecto al viento, presencia de islotes, cercanía al bosque, distancia al mar y altura sobre el nivel del mar. Con ello se realizó un análisis de cluster en base al Índice de Gower con el programa PAST 3.09, lo que permitió agrupar los sitios estudiados acorde a las variables compartidas.

Luego se realizaron análisis de diversidad taxonómica para cada conjunto zooarqueológico para describir la estructura y composición de las faunas. Para estudiar la diversidad dentro de cada sitio se utilizó en primera instancia tres procedimientos estadísticos: riqueza taxonómica (richness), equiparidad (evenness) y heterogeneidad.

La riqueza taxonómica (S) considera el número de taxones identificados, sin mezclar distintos niveles taxonómicos. Es una escala nominal de abundancia taxonómica en base a la presencia/ausencia (Lyman, 2008). La equiparidad taxonómica (E) da cuenta de cómo los individuos se distribuyen entre las categorías, siendo equipar (even) cuando cada taxón presenta el mismo número de individuos y dispar (uneven) cuando cada taxón presenta diferente número de individuos. Se utilizó el Índice de Equiparidad de Shannon: $\mathrm{E}=\mathrm{H} / \operatorname{lnS}$, donde $\mathrm{S}$ corresponde a la riqueza taxonómica y $\mathrm{H}$ a la heterogeneidad. Este indice entrega resultados comprendidos entre el 0 y el 1. El 0 implica que sólo un taxón se encuentra representado, mientras que el 1 señala que todos los taxones son igualmente abundantes, presentando una composición equipar (Nagaoka, 2001; Lyman, 2008). Es importante recordar que este índice no considera la posición u orden de los taxones dentro del conjunto analizado, por lo que hay que tener en cuenta las proporciones relativas de cada taxón.

La heterogeneidad $(\mathrm{H})$ implica una medición simultánea de riqueza y equiparidad. Se midió utilizando el Índice de Shannon-Weaver: $\mathrm{H}=-\sum \mathrm{Pi}(\ln \mathrm{Pi})$, donde $\mathrm{Pi}$ es la proporción del taxón i dentro del conjunto (Lyman, 2008). Los resultados generalmente varían entre 1.5 y 3.5 , siendo más heterogéneos los resultados mayores. Se dividió H por el Logaritmo natural de la riqueza (para cada caso), generando un porcentaje de heterogeneidad, que fue analizado en base a rangos intercuartiles. Consideramos el rango $75 \%-100 \%$ aquel que denota una composición altamente heterogénea.

Luego se dividieron los grupos faunísticos en variables etológicas binarias (peces de aguas someras/peces de aguas demersales; aves solitarias/aves coloniales; mamíferos marinos/ mamíferos terrestres) que fueron correlacionadas con las variables medioambientales por medio del Coeficiente de Correlación de Pearson (r). Cuando el resultado da positivo implica que ambas variables etológicas predominan en un mismo ambiente, y cuando el resultado da negativo implica que las variables etológicas predominan cada una en un ambiente distinto. De este modo se pudo estudiar la influencia del comportamiento de la fauna respecto a su mayor o menor presencia en distintas categorías ambientales. Por último, se compararon los porcentajes relativos de cada taxón en un grupo respecto del otro, evaluando las diferencias en sus proporciones para entender a nivel general las relaciones entre el registro zooarqueológico y los microambientes definidos.

\section{CARACTERIZACIÓN MEDIOAMBIENTAL}

Isla Navarino forma parte de la vertiente sud-pacífico oriental de la Región Archipielágica de Tierra del Fuego (Fig. 1). Se ubica en el sector central del canal Beagle, desde los 54⒌'S con $68^{\circ} 24^{\prime} O$ y tiene una extensión de $2504 \mathrm{~km}^{2}$. Presenta un nudo orográfico situado en el centro 
de la isla, desde el cual drenan más de 20 cursos de agua hacia el canal Beagle, 10 cursos hacia la costa occidental y cuatro hacia la costa sur (Niemeyer \& Cereceda, 1984). Las precipitaciones se distribuyen durante todo el año con promedio de 447,8 mm para Puerto Navarino y 553,4 $\mathrm{mm}$ para Puerto Williams. La temperatura media anual es de $6,1^{\circ} \mathrm{C}$ con oscilación térmica media anual de $7,1^{\circ} \mathrm{C}$ y de $5,5^{\circ} \mathrm{C}$. Hay una marcada humedad hídrica con un promedio de $80 \%$ anual y nubosidad de cielos cubiertos con claros. El promedio anual de vientos es de $12 \mathrm{~km} / \mathrm{hr}$, con rachas máximas de $93 \mathrm{~km} / \mathrm{hr}$, que soplan en dirección sudoeste u oeste (Zamora \& Santana, 1979). La vegetación actual de isla Navarino se compone de Bosque Magallánico Deciduo, Bosque Magallánico Perennifolio, Tundra Magallánica y Desierto Andino (Pisano, 1977). Destacan la lenga, el ñirre, el coigüe y el canelo, el notro y el calafate. Además, hay una amplia variedad de musgos, hongos, hepáticas y líquenes. En la zona litoral hay varias especies de algas (Sielfeld \& Venegas, 1980; Zangrando, 2009).

Se caracterizaron variables ambientales para poder comparar a nivel de sitio los distintos microambientes de manera general. La configuración de las costas quedó determinada a partir del tipo de bahía (abierta o cerrada), la protección de la bahía respecto al viento (protegida, semi protegida o desprotegida) y la presencia/ausencia de islotes. En cuanto a las variables terrestres se determinó la cercanía al bosque a partir de imágenes de Google Earth, al no contar con fotografías o información histórica, y la distancia al mar fue registrada durante las labores de terreno (2010). Estas dos variables fueron luego consideradas en base a rangos de distancia (0-50 m, 50-100 m y > 100 m). No se consideró la hidrografía (cercanía a cursos de agua) ya que la isla Navarino presenta ríos, lagunas y lagos en toda su extensión y son de acceso relativamente fácil desde cualquier sector. Estas características se utilizaron para generar un análisis de cluster en base al Índice de Gower, lo que permitiría eliminar la arbitrariedad en la división de los sitios en dos sectores continuos en el espacio.

De este modo se generaron dos grupos de sitios (Grupo I y Grupo II), ordenados en base a las características medioambientales en común (Tabla 1). El Grupo I incluyó ocho sitios (PN2, BG8, BH14, BH15, BH18, BH26, BM41 y BM42) y el Grupo II, siete sitios (SR32, RL38, BM45, PG56, EV61, BV63 y BV64). Se optó por trabajar con esta división debido a que considera el espacio en

Tabla 1. Características medioambientales asociadas a los sitios.

\begin{tabular}{ccccccc}
\hline Sector & Sitio & $\begin{array}{c}\text { Tipo de } \\
\text { bahía }\end{array}$ & Protección bahía & Islotes & $\begin{array}{c}\text { Cercanía al } \\
\text { bosque }\end{array}$ & $\begin{array}{c}\text { Distancia al mar } \\
\text { (m) }\end{array}$ \\
\hline \multirow{4}{*}{ A } & PN2 & Cerrada & Protegida & sí & $100 \mathrm{~m}$ & 0 a 20 \\
& BG8 & Abierta & Protegida & sí & $0 \mathrm{~m}$ & 40 \\
& BH14 & Cerrada & Protegida & sí & $30 \mathrm{~m}$ & 0 \\
& BH15 & Cerrada & Protegida & sí & $0 \mathrm{~m}$ & 10 \\
& BH18 & Abierta & Protegida & sí & $30 \mathrm{~m}$ & 5 \\
& BH26 & Cerrada & Protegida & sí & $0 \mathrm{~m}$ & 280 y 320 \\
\hline \multirow{4}{*}{ B } & SR32 & Abierta & Desprotegida & no & $50 \mathrm{~m}$ & 30 \\
& RL38 & Abierta & Desprotegida & no & $20 \mathrm{~m}$ & 0 a 10 \\
& BM41 & Cerrada & Semi protegida & sí & $90 \mathrm{~m}$ & 0 a 80 \\
& BM42 & Cerrada & Semi protegida & sí & $80 \mathrm{~m}$ & 1 \\
& BM45 & Cerrada & Desprotegida & no & $40 \mathrm{~m}$ & 0 a 100 \\
& PG56 & Abierta & Desprotegida & no & $0 \mathrm{~m}$ & 85 \\
& EV61 & Cerrada & Semi protegida & no & $0 \mathrm{~m}$ & 10 \\
& BV63 & Abierta & Desprotegida & no & $75 \mathrm{~m}$ & 0 a 50 \\
BV64 & Cerrada & Protegida & no & $0 \mathrm{~m}$ & 0 a 50 \\
\hline
\end{tabular}


función de distintos parches ecológicos semejantes. Es importante destacar que las variables que influyeron mayormente en el resultado de la división de los grupos fueron la presencia de islotes, seguida de aquéllas asociadas a la configuración de las costas (tipo de bahía y protección de bahía). Distancia al mar es la variable que generó menor distinción entre los sitios.

\section{LOS RESTOS ZOOARQUEOLÓGICOS DE ISLA NAVARINO}

La muestra analizada corresponde a un total de 2291 especímenes de restos óseos (NR), de los cuales se pudieron identificar a nivel de género, familia o especie 1042 restos en total (NISP), correspondiendo al $45.48 \%$. Se identificaron aves, peces y mamíferos (Tabla 2 ).

Los restos de los tres grupos faunísticos presentan un buen estado de conservación, con mamíferos presentando meteorización baja en base a los niveles planteados por Behrensmeyer (1978), donde predominan los niveles 0 y 1. Entre los agentes tafonómicos naturales resalta la presencia de radículas, principalmente en los sitios SR32, RL38 y PG56. Otros agentes observados fueron erosión leve, grietas y craquelado, perforaciones y combustión. Además 82 restos presentaron huellas culturales, en su mayoría huellas de corte transversales cortas.

La muestra de peces contiene 332 restos, de los cuales se pudo identificar un total de 159 elementos (47.89\%). Se pudo distinguir siete especies distintas, quedando además una categoría a nivel de familia (Nototheniidae) y dos categorías a nivel de género (Patagonotothen sp. y Merluccius sp.) (Tabla 3).

Esta categoría faunística se encuentra presente en siete de los sitios estudiados. El sitio con mayor abundancia es SR32 con un NISP $=70$, seguido de $\mathrm{BH} 14$ (NISP=48) y PN2 (NISP=24) (Tabla 3).

La muestra de aves contiene 1035 restos, de los cuales se pudo identificar un total de 413 elementos (39.90\%). Se pudo distinguir dos categorías a nivel de familia (Anatidae y Strigiforme), cinco categorías a nivel de género (Chloephaga sp., Phalacrocorax sp., Diomedeidae sp., Ardenna sp. y Larus sp.) y sólo una a nivel de especie (Spheniscus magellanicus). Predominan

Tabla 2. Dimensiones del sondeo por sitio y resumen de restos analizados (NR).

\begin{tabular}{ccccccc}
\hline Sitio & Dimensión $(\mathrm{cm})$ & Profundidad $(\mathrm{cm})$ & Peces & Aves & Mamíferos & Total \\
\hline PN2 & $50 \times 50$ & 110 & 32 & 26 & 26 & 84 \\
BG8 & $50 \times 50$ & 42 & 0 & 15 & 19 & 34 \\
BH14 & $100 \times 50$ & 90 & 87 & 77 & 129 & 293 \\
BH15 & $50 \times 50$ & 20 y 30 & 0 & 0 & 1 & 1 \\
BH18 & $270 \times 50$ & 170 & 5 & 62 & 91 & 158 \\
BH26 & $50 \times 50$ & 40 & 0 & 0 & 19 & 19 \\
SR32 & $50 \times 50$ & 60 & 161 & 190 & 87 & 438 \\
RL38 & $50 \times 50$ & 30 & 0 & 50 & 72 & 122 \\
BM41 & $50 \times 50$ & 60 & 0 & 23 & 9 & 32 \\
BM42 & $50 \times 50$ & 26 & 0 & 9 & 15 & 24 \\
BM45 & $50 \times 50$ & 30 & 0 & 2 & 17 & 19 \\
PG56 & $50 \times 50$ & 20 y 32 & 10 & 451 & 56 & 517 \\
EV61 & $50 \times 50$ & 50 & 6 & 13 & 5 & 24 \\
BV63 & $50 \times 50$ & 90 & 31 & 79 & 287 & 397 \\
BV64 & $50 \times 50$ & 75 & 0 & 38 & 91 & 129 \\
\hline & & 332 & 1035 & 924 & 2291 \\
\hline
\end{tabular}


Tabla 3. Conjunto total de restos de peces identificados taxonómicamente.

\begin{tabular}{|c|c|c|c|c|c|c|c|c|c|c|c|c|c|c|c|c|c|}
\hline Sitio / Taxón & $\underset{\Sigma}{N}$ & $\begin{array}{l}\infty \\
0 \\
\emptyset\end{array}$ & 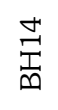 & $\stackrel{\stackrel{L}{D}}{\underset{M}{I}}$ & $\stackrel{\infty}{\underset{\oplus}{\oplus}}$ & $\begin{array}{l}\stackrel{0}{N} \\
\stackrel{T}{ \pm}\end{array}$ & $\begin{array}{l}\text { Nै } \\
\text { Nै } \\
\text { के }\end{array}$ & $\stackrel{\infty}{\stackrel{\infty}{a}}$ & $\sum_{m}^{\vec{F}}$ & $\sum_{\infty}^{\mathcal{N}}$ & $\sum_{\infty}^{\infty}$ & 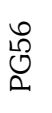 & 常 & $\begin{array}{l}\text { ֶ0 } \\
\text { pे }\end{array}$ & $\begin{array}{l}\nabla_{0} \\
\text { Dे }^{2}\end{array}$ & $\begin{array}{l}0 \\
\text { 号 }\end{array}$ & $\begin{array}{l}\text { कि } \\
\overbrace{0}^{\circ}\end{array}$ \\
\hline Paranotothenia magellanica & 0 & 0 & 0 & 0 & 0 & 0 & 31 & 0 & 0 & 0 & 0 & 0 & 0 & 1 & 0 & 32 & 20.13 \\
\hline Patagonotothen sp. & 0 & 0 & 2 & 0 & 0 & 0 & 11 & 0 & 0 & 0 & 0 & 2 & 0 & 1 & 0 & 16 & 10.06 \\
\hline Nototheniidae indet. & 0 & 0 & 0 & 0 & 1 & 0 & 9 & 0 & 0 & 0 & 0 & 0 & 0 & 0 & 0 & 10 & 6.29 \\
\hline Sebastes oculatus & 1 & 0 & 27 & 0 & 0 & 0 & 2 & 0 & 0 & 0 & 0 & 0 & 0 & 0 & 0 & 30 & 18.87 \\
\hline Cottoperca gobio & 1 & 0 & 1 & 0 & 0 & 0 & 12 & 0 & 0 & 0 & 0 & 0 & 3 & 7 & 0 & 24 & 15.09 \\
\hline Eleginops maclovinus & 1 & 0 & 1 & 0 & 0 & 0 & 0 & 0 & 0 & 0 & 0 & 0 & 0 & 1 & 0 & 3 & 1.89 \\
\hline Thyrsites atun & 0 & 0 & 1 & 0 & 0 & 0 & 0 & 0 & 0 & 0 & 0 & 0 & 0 & 0 & 0 & 1 & 0.63 \\
\hline Salilota australis & 0 & 0 & 2 & 0 & 0 & 0 & 2 & 0 & 0 & 0 & 0 & 0 & 0 & 0 & 0 & 4 & 2.52 \\
\hline Macruronus magellanicus & 20 & 0 & 12 & 0 & 0 & 0 & 0 & 0 & 0 & 0 & 0 & 0 & 0 & 0 & 0 & 32 & 20.13 \\
\hline Merluccius sp. & 1 & 0 & 2 & 0 & 0 & 0 & 3 & 0 & 0 & 0 & 0 & 0 & 1 & 0 & 0 & 7 & 4.40 \\
\hline Total peces & 24 & 0 & 48 & 0 & 1 & 0 & 70 & 0 & 0 & 0 & 0 & 2 & 4 & 10 & 0 & 159 & 100 \\
\hline
\end{tabular}

las aves marinas, siendo Phalacrocorax sp. el taxón más representado (70.22\%), presente en todos los sitios con restos de aves. Sigue en dominancia Diomedeidae sp., con un $17.19 \%$ y luego Spheniscus magellanicus con un $5.81 \%$ (Tabla 4).

Esta categoría faunística se encuentra presente en 13 de los sitios estudiados. Los sitios con mayor abundancia por grupo son SR32 (NISP=70) y PG56 (NISP=184), predominando Diomedeidae sp. en el primero y Phalacrocorax sp. en el segundo. Spheniscus magellanicus se encuentra principalmente en el sitio BV63 $(\mathrm{NISP}=10)$ (Tabla 4).
La muestra de mamíferos contiene 924 restos, de los cuales se pudo identificar un total de 470 elementos (50.87\%). Dentro de los especímenes identificados taxonómicamente se pudo observar la predominancia de la familia Otariidae (74.26\%), seguido de Camelidae (Lama guanicoe, 20.43\%). Además, se pudo distinguir Lontra sp., Cetacea y Canidae (Tabla 5). Los 15 sitios estudiados presentaron esta categoría faunística. Los sitios con mayor abundancia son BH14 (NISP=129) y BV63 (NISP=287), siendo el primero el que presenta mayor cantidad de restos de guanaco y el segundo el que presenta mayor cantidad de Arctocephalus sp. (Tabla 5).

Tabla 4. Conjunto total de restos de aves identificados taxonómicamente.

\begin{tabular}{|c|c|c|c|c|c|c|c|c|c|c|c|c|c|c|c|c|c|}
\hline Sitio / Taxón & ${ }_{\underline{z}}^{N}$ & 怘 & 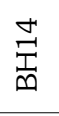 & $\stackrel{\text { 足 }}{\text { 王 }}$ & 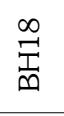 & $\begin{array}{l}\stackrel{0}{N} \\
\underset{\sim}{T}\end{array}$ & $\begin{array}{l}\widetilde{N} \\
\text { कै } \\
\text { ஸै }\end{array}$ & $\begin{array}{l}\stackrel{\infty}{\oplus} \\
\vec{\sim}\end{array}$ & $\sum_{\infty}^{\vec{\sigma}}$ & $\sum_{m}^{\mathcal{N}}$ & $\sum_{\infty}^{L}$ & $\begin{array}{l}\text { ○ } \\
\text { மొ } \\
0 .\end{array}$ & $\begin{array}{l}\overrightarrow{0} \\
\text { 计 }\end{array}$ & 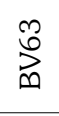 & 莺 & $\frac{n}{n}$ & $\begin{array}{l}\text { कि } \\
\bar{Z} \\
0^{\circ}\end{array}$ \\
\hline Chloephaga sp. & 0 & 0 & 6 & 0 & 0 & 0 & 3 & 0 & 0 & 0 & 0 & 0 & 0 & 0 & 1 & 10 & 2.42 \\
\hline Anatidae indet. & 0 & 0 & 1 & 0 & 0 & 0 & 0 & 0 & 0 & 0 & 0 & 0 & 0 & 1 & 0 & 2 & 0.48 \\
\hline Phalacrocorax sp. & 2 & 1 & 19 & 0 & 32 & 0 & 7 & 13 & 8 & 2 & 1 & 179 & 2 & 8 & 16 & 290 & 70.22 \\
\hline Diomedeidae sp. & 7 & 0 & 0 & 0 & 0 & 0 & 50 & 2 & 0 & 1 & 0 & 1 & 3 & 4 & 3 & 71 & 17.19 \\
\hline Ardenna sp. & 0 & 0 & 1 & 0 & 0 & 0 & 4 & 1 & 0 & 0 & 0 & 0 & 0 & 5 & 0 & 11 & 2.66 \\
\hline Larus sp. & 1 & 0 & 0 & 0 & 0 & 0 & 0 & 1 & 0 & 0 & 0 & 2 & 0 & 0 & 0 & 4 & 0.97 \\
\hline Spheniscus magellanicus & 0 & 0 & 2 & 0 & 2 & 0 & 6 & 0 & 2 & 0 & 0 & 2 & 0 & 10 & 0 & 24 & 5.81 \\
\hline Strigiformes indet. & 0 & 0 & 0 & 0 & 1 & 0 & 0 & 0 & 0 & 0 & 0 & 0 & 0 & 0 & 0 & 1 & 0.24 \\
\hline Total aves & 10 & 1 & 29 & 0 & 35 & 0 & 70 & 17 & 10 & 3 & 1 & 184 & 5 & 28 & 20 & 413 & 100 \\
\hline
\end{tabular}




\section{FAUNA Y MICROAMBIENTES}

Una vez realizado el análisis zooarqueológico se procedió a evaluar el grado de equiparidad que presentan los conjuntos de la costa noroeste de isla Navarino. Para ello se consideró el NISP, descontando algunos taxones que generan interdependencia entre sí, con ello el número total de restos identificados fue de 971. Se realizó un análisis de diversidad taxonómica considerando riqueza, equiparidad, heterogeneidad y porcentaje de heterogeneidad.
Es importante recordar que estos índices se encuentran fuertemente influenciados por el tamaño de la muestra. Por lo mismo se aplicó el Coeficiente de Correlación de Pearson (r) y el Coeficiente de Determinación $\left(\mathrm{r}^{2}\right)$, contrastando el NISP con los distintos índices calculados. Como resultado se observó que sólo la riqueza se encuentra medianamente determinada por el tamaño de la muestra (67\%), mientras que la equiparidad y la heterogeneidad presentan una baja determinación (14\%). De igual manera creímos conveniente considerar solamente los sitios que presenten un

Tabla 5. Conjunto total de restos de mamíferos identificados taxonómicamente.

\begin{tabular}{|c|c|c|c|c|c|c|c|c|c|c|c|c|c|c|c|c|c|}
\hline Sitio / Taxón & 兄 & $\begin{array}{l}\infty \\
\bigcup_{\varphi}\end{array}$ & 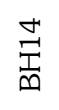 & 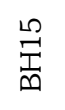 & $\stackrel{\infty}{\stackrel{\infty}{I}}$ & $\begin{array}{l}\stackrel{\vartheta}{N} \\
\stackrel{\underline{I}}{\oplus}\end{array}$ & $\begin{array}{l}\tilde{N} \\
\tilde{\omega} \\
\tilde{\omega}\end{array}$ & $\stackrel{\infty}{\stackrel{\infty}{*}}$ & $\sum_{\dot{m}}^{\vec{g}}$ & $\sum_{\infty}^{\mathcal{N}}$ & $\sum_{\infty}^{\stackrel{L}{\infty}}$ & $\begin{array}{l}\text { ڤ } \\
\text { L }\end{array}$ & $\begin{array}{l}\vec{b} \\
\vec{u}\end{array}$ & 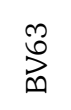 & $\begin{array}{l}\overrightarrow{0} \\
\text { 命 }\end{array}$ & 命 & $\begin{array}{l}\stackrel{0}{Z^{\circ}} \\
\delta^{\circ}\end{array}$ \\
\hline Otaria flavescens & 0 & 0 & 1 & 0 & 0 & 1 & 0 & 0 & 0 & 0 & 0 & 3 & 0 & 1 & 0 & 6 & 1.28 \\
\hline Arctocephalus sp. & 12 & 4 & 7 & 1 & 11 & 17 & 37 & 24 & 0 & 2 & 2 & 26 & 0 & 131 & 17 & 291 & 61.91 \\
\hline Otariidae indet. & 3 & 5 & 4 & 0 & 1 & 0 & 5 & 2 & 4 & 0 & 2 & 0 & 0 & 21 & 5 & 52 & 11.06 \\
\hline Lontra sp. & 0 & 0 & 0 & 0 & 1 & 0 & 1 & 4 & 0 & 1 & 0 & 3 & 0 & 4 & 0 & 14 & 2.98 \\
\hline Cetacea indet. & 0 & 0 & 0 & 0 & 6 & 0 & 0 & 0 & 0 & 0 & 1 & 0 & 0 & 2 & 0 & 9 & 1.91 \\
\hline Lama guanicoe & 0 & 4 & 33 & 0 & 16 & 0 & 3 & 13 & 0 & 8 & 9 & 2 & 3 & 3 & 2 & 96 & 20.43 \\
\hline Canidae indet. & 0 & 0 & 1 & 0 & 1 & 0 & 0 & 0 & 0 & 0 & 0 & 0 & 0 & 0 & 0 & 2 & 0.43 \\
\hline Total mamíferos & 15 & 13 & 46 & 1 & 36 & 18 & 46 & 43 & 4 & 11 & 14 & 34 & 3 & 162 & 24 & 470 & 100 \\
\hline
\end{tabular}

Tabla 6. Análisis de diversidad taxonómica para cada sitio. Riqueza (S), equiparidad (E), heterogeneidad $(\mathrm{H})$ y porcentaje de heterogeneidad $(\% \mathrm{H})$. El gris señala los sitios con NISP > 50

\begin{tabular}{ccccc}
\hline Sitio & S & E & H & $\% \mathrm{H}$ \\
\hline PN2 & 15 & 0.66 & 2.01 & 74.09 \\
BG8 & 9 & 0.49 & 1.48 & 67.37 \\
BH14 & 14 & 0.65 & 1.99 & 75.30 \\
BH15 & 7 & 0.48 & 1.48 & 75.85 \\
BH18 & 9 & 0.24 & 0.73 & 33.35 \\
BH26 & 14 & 0.38 & 1.16 & 43.98 \\
SR32 & 8 & 0.49 & 1.48 & 71.13 \\
RL38 & 3 & 0.32 & 0.96 & 87.83 \\
BM41 & 1 & 0 & 0 & 0 \\
BM42 & 2 & 0.07 & 0.21 & 30.95 \\
BM45 & 3 & 0.16 & 0.50 & 45.55 \\
PG56 & 5 & 0.41 & 1.25 & 77.84 \\
EV61 & 4 & 0.31 & 0.94 & 67.60 \\
BV63 & 5 & 0.45 & 1.37 & 85.31 \\
BV64 & 5 & 0.38 & 1.17 & 72.76 \\
\hline
\end{tabular}


NISP > 50 (BH14, BH18, SR32, RL38, PG56 y BV63).

Al observar los resultados respecto a estos seis sitios, se da cuenta que hay dos sitios que presentan una estructura más equipar y heterogénea: $\mathrm{BH} 14$ y SR32. Éstos coinciden con ser aquéllos sitios que presentan mayores valores para restos de peces. Por su parte, PG56 y BV63 presentan valores más bajos para ambos índices, comportándose de manera dispar y más homogénea. Estos dos sitios presentan gran cantidad de restos de cormoranes y de pinnípedos respectivamente, denotando predominancia de un recurso por sobre los demás. Los otros dos sitios, BH18 y RL38, presentan resultados intermedios para ambas variables, siendo ambos medianamente heterogéneos y equipares en cuanto a su composición faunística.

De estos seis sitios sólo dos pertenecen al Grupo I, mientras que los demás pertenecen al Grupo II. Así, el primer grupo presentaría un sitio heterogéneo (BH14) y uno medianamente heterogéneo (BH18), y el segundo grupo presentaría un sitio heterogéneo (SR32), uno medianamente heterogéneo (RL38) y dos sitios homogéneos (PG56 y BV63).

Esto implica diferencias en el espacio respecto a la presencia y proporciones de los recursos, lo que puede deberse a diversos factores. Por ello se estudió cómo se comportan las variables de diversidad taxonómica respecto a los dos grupos medioambientalmente determinados, considerando todos los sitios analizados. Como resultado se observa que ambos grupos son medianamente equipares y heterogéneos, como se esperaría en un contexto donde los recursos se encuentran distribuidos de manera relativamente homogénea en el espacio. Esto se asocia a la dieta diversificada que caracteriza a los grupos canoeros con adaptación litoral. Sin embargo, el Grupo I parece ser sutilmente más heterogéneo y equipar que el Grupo II (Tabla 7). Ello se corresponde con la presencia en el segundo grupo de los dos sitios cuyos conjuntos faunísticos se comportan de manera homogénea y dispar (PG56 y BV63).

Lo anterior estaría evidenciando pequeñas diferencias en la estructura y composición de las faunas en el espacio y entre ambos grupos. Para evaluar la influencia de las variables ambientales en la composición del registro zooarqueológico se optó por dividir cada categoría faunística en base a características etológicas binarias y que constituyesen diferenciación en el uso del espacio. De este modo consideramos peces de aguas someras y de aguas demersales, aves de comportamiento solitario o que forman colonias, y mamíferos marinos y terrestres.

A partir de ello asociamos el tipo de comportamiento con las variables ambientales, estimando un Coeficiente de Correlación ( $\mathrm{r}$ ) en base al NISP. Así pudimos agrupar dos conjuntos de variables, ambiental y etológica, y asociarlas a los Grupos de sitios ya definidos (Tabla 8). El caso de las aves que conforman colonias queda intermedio entre ambos grupos, aunque asemejándose más

Tabla 7. Análisis de diversidad taxonómica para cada grupo. Número de restos (NR), número de especímenes identificados considerados (NISP), riqueza (S), equiparidad (E), heterogeneidad (H) y porcentaje de heterogeneidad (\% H).

\begin{tabular}{cccccccc}
\hline Grupo & NR & NISP & S & E & H & \% H \\
\hline I & 645 & 283 & 20 & 0.7 & 2.13 & 71.07 \\
\hline II & 1646 & 688 & 17 & 0.6 & 1.81 & 64.00 \\
\hline
\end{tabular}

Tabla 8. Relación entre variables etológicas y variables ambientales y su correspondencia con los grupos definidos.

\begin{tabular}{ccc}
\hline Variable ambiental & Variable etológica & Grupo \\
\hline Bahía cerrada & Peces de aguas demersales & \\
Bahía protegida & Mamíferos terrestres & I \\
Presencia de islotes & & \\
Distancia al bosque 0-50 $\mathrm{m}$ & Peces de aguas someras & II \\
Bahía abierta & Mamíferos marinos & Aves solitarias \\
Bahía desprotegida & Aves coloniales & \\
Ausencia de islotes & & \\
Distancia al bosque $50-100 \mathrm{~m}$ & &
\end{tabular}


al segundo. Al observar cómo se comportan estos dos conjuntos dentro de los grupos destacamos que en el primero hay mayor presencia de Macruronus magellanicus, Sebastes oculatus y Lama guanicoe. Mientras, en el segundo grupo hay mayor presencia de Paranotothenia magellanica y Diomedeidae sp.

Sin embargo, si observamos las proporciones relativas de las características etológicas dentro de cada grupo, se evidencia que los mamíferos marinos predominan en el Grupo I a pesar de presentar menores cantidades que en el Grupo II, donde predominan las aves que conforman colonias (Tabla 9).

\section{DISCUSIÓN}

Buscando comprender lasvariablesespaciales que pueden influir en la selección de recursos faunísticos, realizamos un trabajo exploratorio en el que se caracterizaron microambientes y se estudiaron los conjuntos zooarqueológicos de diferentes pozos de sondeo a lo largo de la costa noroeste de isla Navarino. De este modo nuestro trabajo pretende discutir las posibles relaciones que se presentan entre las variables microambientales $y$ el registro zooarqueológico.

El Modelo de Elección de Parcelas plantea la división del espacio en parches ecológicos que sean homogéneos en cuanto a las posibilidades de encontrar determinados recursos (Nagaoka, 2001), es decir, que presenten los mismos costos de búsqueda. Considerando la similitud entre los ambientes de isla Navarino, buscamos dividir el área de estudio en dos conjuntos que pudiesen ser diferenciables geomorfológica y ambientalmente (Grupo I y Grupo II) y que, por ende, pudiesen presentar diferencias en los costos de obtención de los recursos. Estos conjuntos no son continuos en el territorio sino que se presentan de manera intercalada.

Las variables ambientales que mayormente influyeron en la determinación de los grupos microambientales fueron aquellas asociadas a la configuración de las costas (tipo de bahía, protección de bahía y presencia de islotes). Hay variables que no pudimos utilizar en el contexto de este trabajo y que consideramos sumamente relevantes para entender las diferencias en el acceso a los recursos. Entre ellas están el tipo de submareal y fondo marino, que influyen en la presencia/ausencia de distintas especies de peces (Moreno \& Jara, 1984) y de moluscos (Piana \& Orquera, 2010). Entendemos que asociar estas variables a los sitios estudiados permitiría acercarnos de manera más precisa a la selección de recursos ictiológicos en base a su disponibilidad en el ambiente. Además, sería interesante estudiar las pendientes que presenta el talud costero en los distintos sitios, ya que las costas con menor angularidad favorecerían la posibilidad de varamientos masivos de algunas especies (Zangrando, 2009); por ejemplo, Macruronus magellanicus, especie de las más representadas en el registro analizado, que tiende a generar grandes varamientos en el período estival los cuales suelen ocurrir en costas con menor pendiente (Lloris \& Rucabado, 1991).

Las variables etológicas seleccionadas en este trabajo para mamíferos y peces son efectivamente discriminatorias en cuanto a los ambientes preferidos por las distintas especies. Para el caso de las aves consideramos que la variable de comportamiento colonial o solitario no cumple a cabalidad con la diferenciación

Tabla 9. NISP y proporciones relativas de las características etológicas respecto a los grupos determinados ambientalmente.

\begin{tabular}{lcccc}
\hline \multicolumn{1}{c}{ Etología } & Grupo I & \% Grupo I & Grupo II & \% Grupo II \\
\hline Peces de aguas someras & 4 & 1.20 & 68 & 11.53 \\
Peces de aguas demersales & 60 & 18.07 & 2 & 0.34 \\
Aves: solitarias/parejas & 14 & 4.22 & 67 & 11.36 \\
Aves: colonias & 70 & 21.08 & 244 & 41.36 \\
Mamíferos marinos & 123 & 37.05 & 174 & 29.49 \\
Mamíferos terrestres & 61 & 18.37 & 35 & 5.93 \\
\hline \multicolumn{1}{c}{ Total } & 332 & 100 & 590 & 100 \\
\hline
\end{tabular}


ambiental propuesta, principalmente porque aves de ambas características se pueden encontrar muchas veces utilizando los mismos espacios. Aves de comportamiento colonial, además, no necesariamente se encuentran todo el tiempo en grupos. Consideramos que esta distinción resulta útil al evaluar las proporciones de las diferentes especies de aves, entendiendo que aquellas especies que conforman colonias pueden ser cazadas de manera masiva (Tivoli, 2010). En relación a ello resulta necesario conocer la ubicación de posibles sectores de nidificación de estas especies y sus distancias a los sitios estudiados. Para las aves, entonces, habría que evaluar otra variable que permita distinguir etológica y ambientalmente a las especies, por ejemplo, si son aves terrestres, litorales o pelágicas.

\section{Equiparidad}

Los análisis de diversidad taxonómica dieron cuenta de sitios equipares, dispares y sitios con equiparidad cercana a 0.5 . Esto implica diferencias en el espacio respecto a la presencia y proporciones de los recursos faunísticos, lo que puede deberse a diferentes factores. Un factor interesante a discutir es la temporalidad de los sitios. Para la costa norte del canal Beagle se plantea un cambio en los patrones de dieta entre los sitios más tempranos y los más tardíos (Tivoli \& Zangrando, 2011). A partir del análisis de diversidad realizado a cinco sitios se obtuvo un incremento moderado en la equiparidad de 0.55 en Imiwaia I (K, L y M) a 0.68 en Shamakush X. Se señaló que el incremento en la equiparidad era significativo $(r=0.84, p<0.05)$, lo que se interpretó como cambios en la subsistencia, aunque manteniendo una economía diversificada. Esto se relaciona a un continuo decrecimiento en las abundancias de pinnípedos ligado a un progresivo aumento en las proporciones de aves y peces (Zangrando, 2009; Tivoli, 2010; Tivoli \& Zangrando, 2011). Estos datos pueden servir de expectativas para la equiparidad y su relación con el comportamiento temporal de los conjuntos.

Resulta importante mencionar que los sitios considerados en los trabajos realizados para la costa norte del canal Beagle presentan un solo bloque temporal, a excepción de Imiwaia I (Tivoli \& Zangrando, 2011). Ello implica que se están evaluando cambios en el tiempo considerando conjuntos zooarqueológicos ubicados en diferentes lugares en el espacio, y no en base a expresiones estratigráficas. Por ello, consideramos relevante, desde un punto de vista comparativo, incluir en la discusión sobre la equiparidad la influencia de las distintas variables microambientales inmediatas a los sitios trabajados y su influencia respecto a la disponibilidad y acceso a los recursos faunísticos.

Por lo mismo, se evaluó la influencia de distintas variables ambientales en el registro zooarqueológico. Generamos dos grupos microambientales y evaluamos la equiparidad para dichos conjuntos. Ambos resultaron ser medianamente equipares y heterogéneos, lo que se relaciona con la dieta diversificada que caracteriza a los grupos canoeros con adaptación litoral (Orquera \& Piana, 1999; Legoupil et al. 2011). Sin embargo, el Grupo I es sutilmente más equipar y heterogéneo, lo que se relaciona a la presencia de los sitios con conjuntos homogéneos PG56 y BV63 en el Grupo II. Entendemos que la división espacial generada no necesariamente se correspondería con una división temporal (sitios tempranos / sitios tardíos), de hecho, sitios que por el momento pueden ser adscritos al Holoceno tardío se encuentran presentes en ambos grupos. Más bien suponemos que esta diferencia en la equiparidad se asocia al acceso a los diferentes recursos que generan las variables ambientales inmediatas a los sitios, asociado a la etología y el comportamiento de la fauna. Así, los sitios más protegidos y con bahías cerradas presentarían una disponibilidad de recursos que genera conjuntos más heterogéneos y diversos. Mientras, en las bahías abiertas y desprotegidas se generaron conjuntos más homogéneos, con predominancia de unos recursos por sobre otros.

Nos parece pertinente poder evaluar a futuro, con una mayor cantidad de datos, una conjunción entre las variables espacial y temporal. De ese modo buscar comprender de manera completa la disponibilidad y selección de recursos faunísticos en el canal Beagle.

\section{Criterios de selección}

Para estudiar los criterios de selección partimos de la Ecología del Comportamiento 
Humano y sus modelos sobre forrajeo óptimo. Éstos plantean que las elecciones de recursos a seleccionar están basadas en la búsqueda del mayor retorno energético por el menor esfuerzo y riesgo (Perlman, 1980). A partir de esta idea se pueden generar predicciones respecto a las elecciones humanas (Kelly, 2013).

El Modelo de Amplitud de Dieta considera los costos de búsqueda, manejo y rangos de retorno de los recursos para generar un ranking que permite predecir qué tan ancha o angosta será la dieta de un determinado grupo humano (Neusius, 1986; Kelly, 2013). Un cazador preferiría siempre los recursos de mayor ranking, por lo que la inclusión de recursos de menor rango depende de la disponibilidad y abundancia de los recursos preferidos (Kelly, 2013). Así, la dieta será angosta cuando hay una alta disponibilidad de recursos de alto rango, generando conjuntos heterogéneos enfocados en dichos recursos. Cuando hay menor disponibilidad de ellos aumenta la probabilidad de seleccionar recursos de menor rango, por lo que la dieta será más ancha (amplia) y los conjuntos más homogéneos (Neusius, 1986). Se ha planteado que en casos arqueológicos los recursos se valorizan en función del tamaño-peso corporal, la abundancia relativa, el potencial de explotación masiva y las estrategias tecnológicas (Zangrando, 2009).

Dado que no profundizamos en los costos y rangos de retorno energético, nos enfocamos en la disponibilidad y abundancia de recursos en el espacio. Para ello estudiamos las variables medioambientales que influyen en la presencia de las diferentes especies. Recordemos que los grupos cazadores-recolectores con una organización forrajera se caracterizan por un sistema de subsistencia y asentamiento basado en la recolección diaria de recursos y una movilidad residencial (Binford, 1980). Esto sería posible gracias a que la distribución de los recursos se da de manera homogénea o constante en el territorio. Sin embargo, observamos pequeñas diferencias entre los distintos lugares de asentamiento, y las decisiones de recolección diaria cambiarian, generando diferentes estructuras en el registro zooarqueológico.

La variabilidad observada en los conjuntos zooarqueológicos estaría dando cuenta de elecciones humanas de selección. Consideramos que éstas representan las elecciones adoptadas en función de los diferentes microambientes y disponibilidades de los recursos. Como ya hemos mencionado, los sitios más protegidos y con bahías cerradas presentarían una disponibilidad de recursos que genera conjuntos más heterogéneos y diversos. Mientras, en las bahías abiertas y desprotegidas se generaron conjuntos más homogéneos, con predominancia de unos recursos por sobre otros. Esto se condice con las expectativas propuestas entre las características del medio ambiente y la expectativa de diversidad taxonómica. Sin embargo, en cuanto a las expectativas del registro zooarqueológico hubo discrepancias con lo observado.

Profundizamos entonces en las elecciones de recursos en función de los microambientes planteados. En los dos grupos observamos que los dos recursos predominantes son pinnípedos y cormoranes. Sin embargo, en el Grupo II preponderan los cormoranes, lo que nos hace suponer que los costos de obtención del pinnípedo pueden ser mayores en estos microambientes. A partir de ello uno podría inferir una mayor presencia de pinnípedos en bahías cerradas y protegidas, así como mayor abundancia de cormoranes en bahías abiertas y desprotegidas.

Finalmente es importante mencionar que hay otras variables a considerar en el proceso de selección de recursos ya que no siempre se opta por las decisiones más eficientes y maximizadoras. Es necesario tener en cuenta entonces variables como el sabor, el prestigio, los tabúes y la valorización que se le da a cada recurso (Fiore \& Zangrando, 2006; Zangrando, 2009; Stambuk, 2011).

\section{COMENTARIOS FINALES}

Este trabajo consistió en una búsqueda preliminar de variables espaciales que influyen en la estructura de los conjuntos zooarqueológicos. Consideramos que la división del espacio realizada da cuenta de variabilidad dentro de un ambiente homogéneo, y se presenta como un buen inicio para empezar a buscar aquellas diferencias. Sin embargo, se requiere precisar algunas de las variables trabajadas e identificar nuevas variables que aporten mayor información contextual para la diferenciación de los sitios.

Pese a que la muestra es pequeña pudimos observar que hay sitios que se comportan de 
manera diferente. Aunque comprendemos la necesidad de ampliar las muestras para saber si las tendencias propuestas son representativas del área de estudio en general.

Resulta indispensable generar fechados radiocarbónicos para evaluar la influencia de las diferencias temporales entre los conjuntos y poder comparar esta variable con los resultados planteados en la costa norte del canal Beagle. Además, conocer la temporalidad nos permitirá acercarnos mejor a la configuración de los microambientes del pasado.

La diversidad espacial y temporal observada para ambas costas del canal Beagle contrasta con la continuidad y estabilidad en los patrones generales de subsistencia en más de 6.000 años de ocupación, planteados por Orquera y Piana. Por ello consideramos relevante poder seguir avanzando en esta temática para identificar y evaluar nuevas variables etológicas y microambientales que nos permitan acercarnos cada vez más a las pautas de subsistencia humana.

Sería interesante a futuro poder generar un modelo predictivo que permita reconocer cuándo la composición de los sitios zooarqueológicos no se comporta acorde a lo esperado en base a las características ecológicas y ambientales, lo que implicaría posibles causas culturales que deberían ser estudiadas en cada contexto.

\section{AGRADECIMIENTOS}

Esta investigación ha sido financiada por el proyecto FONDECYT 1140939. Agradezco a César Méndez por su guía y apoyo, a Flavia Morello por invitarme a participar en su proyecto, a Manuel San Román, Jimena Torres, Ismael Martínez, Angélica Tivoli, Francisco Zangrando y a todos los que me ayudaron resolviendo dudas que iban surgiendo en el camino.

\section{BIBLIOGRAFÍA}

Behrensmeyer, A. (1978). Taphonomic and ecologic information from bone weathering. Paleobiology, 4(2), 150-162.

Binford, L. (1980). Willow smoke and dog's tails: huntergatherer settlement systems and archaeological site formation. American Antiquity, 45(1), 4-20.

Fiore, D., \& Zangrando, A. F. (2006). Painted fish, eaten fish:
Artistic and archaeofaunal representations in Tierra del Fuego, Southern South America. Journal of Anthropological Archaeology, 25, 371-389.

Kelly, R. (2013). The Lifeways of Hunter-Gatherers: The Foraging Spectrum (2a ed.). Washington y London: Smithonian Institution Press.

Legoupil, D., \& Fontugne, M. (1997). El poblamiento marítimo en los archipiélagos de Patagonia: núcleos antiguos y dispersión reciente. Anales del Instituto de la Patagonia, 25, 75-87.

Legoupil, D., Béarez, P., Lefévre, C., San Román, M., \& Torres, J. (2011). Estrategias de subsistencia de cazadores recolectores de Isla Dawson (Estrecho de Magallanes) durante la segunda mitad del Holoceno: primeras aproximaciones. Magallania, 39(2), 153-164.

Lloris, D., \& Rucabado, J. (1991). Ictiofauna del canal Beagle (Tierra del Fuego): aspectos ecológicos y análisis biogeográfico. Madrid, España: Instituto Español de Oceanografía.

Lyman, R. (2008). Quantitative Paleozoology. Cambridge Manuals in Archaeology. Oxford, Inglaterra: Cambridge University Press.

Morello, F., Borrero, L., Massone, M., Stern, C., GarcíaHerbst, A., \& Mc Culloch, R. (2012). Hunter-gatherers, biogeographic barriers and the development of human settlement in Tierra del Fuego. Antiquity, 86, 71-87.

Moreno, C., \& Jara, F., (1984). Ecological studies on fish fauna associated with Macrocystis pyrifera belts in the south of Fueguian Islands, Chile. Marine Ecology - Progress series, 15, 99-107.

Nagaoka, L. (2001). Using diversity indices to measure changes in prey choice at the Shang River Mouth Site, Southern New Zealand. International Journal of Ostoearchaeology, 11, 101-111.

Neusius, S. (1986). Generalized and specialized resource utilization during the Archaic Period: implications of the Koster site faunal record. En S. Neusius (Ed.), Foraging, collecting and harvesting: Archaic period subsistence and settlement in the eastern woodlands (pp. 117-143). Illinois, IL: Center of Archaeological Investigations Occasional Paper 6: Board of Trustees Southern Illinois University.

Niemeyer, H., \& Cereceda, P. (1984). Geografía de Chile. Tomo VIII Hidrografía. Santiago de Chile: Instituto Geográfico Militar.

Orquera, L., \& Piana, E. (1999). Arqueología de la región del canal Beagle (Tierra del Fuego, República Argentina). Buenos Aires, Argentina: Sociedad Argentina de Antropología. 
Orquera, L., \& Piana, E. (2009). Sea Nomads of the Beagle Channel in Southernmost South America: Over Six Thousand Years of Coastal Adaptation and Stability. Journal of Island and Coastal Archaeology, 4, 61-81.

Orquera, L., Legoupil, D., \& Piana, E. (2011). Littoral adaptation at the southern end of South America. Quaternary International, 239, 61-69.

Perlman, S. (1980). An optimum diet model, coastal variability, and hunter-gatherer behaviour. En M. Schiffer (Ed.), Advances in archaeological method and theory (Vol. 3, pp. 257-309). USA: Academic Press.

Piana, E., \& Orquera, L. (2010). Shellmidden formation at the Beagle Channel (Tierra del Fuego, Argentina). En D. Calado, M. Baldía \& M. Boulanger (Eds.), Monumental questions: Prehistoric megaliths, mounds, and enclosures (pp. 263-271). Oxford, Inglaterra: BAR International Series.

Piana, E., Vásquez, M., \& Tivoli, A. (2007). Dieta y algo más. Animales pequeños y variabilidad del comportamiento humano en el Canal Beagle. En F. Morello, M. Martinic, A. Prieto, y G. Bahamondes (Eds.), Levantando piedras, desenterrando huesos... y develando arcanos (pp. 39-50). Punta Arenas: Ediciones CEQUA.

Pisano, E. (1977). Fitogeografía de Fuego-Patagonia Chilena. I.- Comunidades vegetales entre las Latitudes $52^{\circ}$ y $56^{\circ}$ S. Anales del Instituto de la Patagonia, 8, 121-250.

San Román, M. (2011). La explotación de recursos faunísticos en el sitio Punta Santa Ana 1: estrategias de subsistencia de grupos de cazadores marinos tempranos de Patagonia Meridional. Tesis de pregrado. Universidad de Chile, Santiago, Chile.

Sielfeld, W., \& Venegas, C. (1980). Poblamiento e impacto ambiental de Castor canadensis kuhl, en isla Navarino. Chile. Anales del Instituto de la Patagonia, 11, 247257.

Stambuk, P. (2011). Rosa Yagán. Lakutaia Le Kipa (7a ed.). Biblioteca del Bicentenario. Chile: Pehuén.

Tivoli, A. (2010). Las aves en la organización socioeconómica de cazadores-recolectores-pescadores del Extremo Sur Sudamericano. Buenos Aires, Argentina: Sociedad Argentina de Antropología.

Tivoli, A., \& Zangrando, A. F. (2011). Subsistence variations and landscape use among maritime hunter-gatherers. A zooarchaeological analysis from the Beagle Channel (Tierra del Fuego, Argentina). Journal of Archaeological Science, 38, 1148-1156.

Zamora, E., \& Santana, A. (1979). Características climáticas de la costa occidental de la Patagonia entre las Latitudes $46^{\circ} 40^{\prime}$ y $56^{\circ} 30^{\prime} \mathrm{S}$. Anales del Instituto de la Patagonia, 10, 109-144.

Zangrando, A. F. (2009). Historia evolutiva y subsistencia de cazadores-recolectores marítimos de Tierra del Fuego. Buenos Aires, Argentina: Sociedad Argentina de Antropología. 